\title{
THE DIAGNOSIS OF TRICUSPID STENOSIS
}

BY

\author{
RONALD GIBSON AND PAUL WOOD \\ From the Cardiac Department of the Brompton Hospital and the Institute of Cardiology
}

Received April 20, 1955

The clinical features of tricuspid stenosis have never been clearly defined. The diagnosis has usually been made in the late stages of the disease or it has only been revealed at necropsy. According to Friedberg (1949), the signs of tricuspid stenosis include accentuation of the $a$ wave of the jugular pulse in normal rhythm, a diastolic or presystolic murmur at the left sternal edge, and enlargement of the right atrium radiologically; and tricuspid valve disease is suggested by a pulsating liver and a positive systolic venous pulse in a patient with systemic venous congestion, cyanosis, ascites, and valvular heart disease. White (1951) considers that the tricuspid diastolic murmur is difficult to distinguish from the murmur of mitral stenosis transmitted from the apex, and describes enlargement of the right atrium with a prominent systolic jugular pulse, in the absence of irreversible tricuspid ring dilatation as corroborative signs of tricuspid stenosis. Bramwell and King (1942) emphasized the powerful presystolic wave in the jugular pulse and the tall $\mathrm{P}$ wave in the electrocardiogram in cases of tricuspid stenosis. Kossman (1955) has drawn attention to the tricuspid opening snap. Reviews of necropsy material show that the diagnosis is made in only 10 to 15 per cent of cases (Cooke and White, 1941; Smith and Levine, 1942). Ferrer et al. (1953) state that the clinical diagnosis of tricuspid stenosis is unsatisfactory and is possible only in advanced cases.

Although rheumatic tricuspid stenosis is rare and its clinical features are usually over-shadowed by those of the concomitant mitral valve lesion, it is our belief that the diagnosis is important for the following reasons: it may masquerade as heart failure; it always modifies the clinical features of mitral stenosis and accounts for certain signs that, if attributed to the mitral lesion, would give a false impression of the physiological situation; and rarely it is the dominant lesion and may be severe enough to warrant valvotomy.

The purpose of this paper is to clarify the criteria upon which a confident diagnosis of tricuspid stenosis can be made during life, both at the bedside and by means of certain routine investigations, and whether the lesion is mild or advanced. It is based on a study of fifteen cases, and constitutes an elaboration and extension of the criteria outlined by one of us previously (Wood, 1954).

\section{MATERIAL}

There were fourteen cases of rheumatic mitral stenosis with tricuspid stenosis, nine of them occurring in a consecutive series of three hundred cases of mitral valve disease studied in detail by Wood (1954), an incidence of 3 per cent. Although tricuspid stenosis modified the clinical features of the associated mitral valve disease in all cases, it was the dominant lesion in only three. Six cases had trivalvular disease, there being an additional aortic lesion in five and pulmonary stenosis in one.

A single case of isolated tricuspid stenosis in a patient with presumed disseminated lupus erythematosus will be described separately. 


\section{Clinical Features of the Rheumatic Cases}

Age and Sex. There were twelve women and two men, their ages ranging from 20 to 48 and averaging 36 years.

Antecedent Rheumatic Infection. There was a previous history of rheumatic fever in eight cases and of chorea in two.

Symptoms. The average age at the onset of symptoms was 31 years. Effort intolerance was considerable in six cases, moderate in six, and slight in two. Orthopnœa and paroxysmal cardiac dyspnœa occurred in only three instances, and both pulmonary apoplexy and congestive hæmoptysis, as defined elsewhere (Wood, 1954), were conspicuous by their absence.

The first symptom in two cases of dominant tricuspid stenosis was fluttering in the neck, especially on effort, due to the early development of giant $a$ waves; in one case it preceded other symptoms by ten years.

Levine and Thompson (1937) emphasized that the ability to lead a fairly active life despite recurrent œdema and ascites was characteristic of tricuspid stenosis, and Smith and Levine (1942) found that the average duration of life under these circumstances was $7 \cdot 5$ years. Three of the more advanced cases in this series behaved in this manner.

Jugular Venous Pressure and Pulse. Presystolic pulsation in the jugular pulse in tricuspid stenosis was described by Mackenzie (1902). All eight cases with normal rhythm in this series showed a giant $a$ wave in the jugular pulse ranging from 4 to $10 \mathrm{~cm}$. above the sternal angle (Fig. 1); this giant $a$ wave provided excellent evidence of tricuspid stenosis when there were no other signs of an extreme pulmonary vascular resistance. In these eight cases $v$ was insignificant except in one instance complicated by congestive heart failure secondary to mitral stenosis with pulmonary hypertension.

In five of the six cases with auricular fibrillation, the venous pressure ranged between 6 and $10 \mathrm{~cm}$. above the sternal angle and the venous pulse was systolic. This high venous pressure proved good evidence of tricuspid stenosis provided three conditions were fulfilled; namely, that the ventricular rate was controlled, that there were no other signs of a high pulmonary vascular resistance, and that the mitral valve was stenosed rather than incompetent.

The form of the venous pulse in tricuspid stenosis is characteristic even when there is auricular fibrillation and no giant $a$ wave, for the $y$ descent is usually too gentle to admit of any other diagnosis. When the venous pressure is high in other conditions, the downstroke of $v$ ( $y$ descent) is

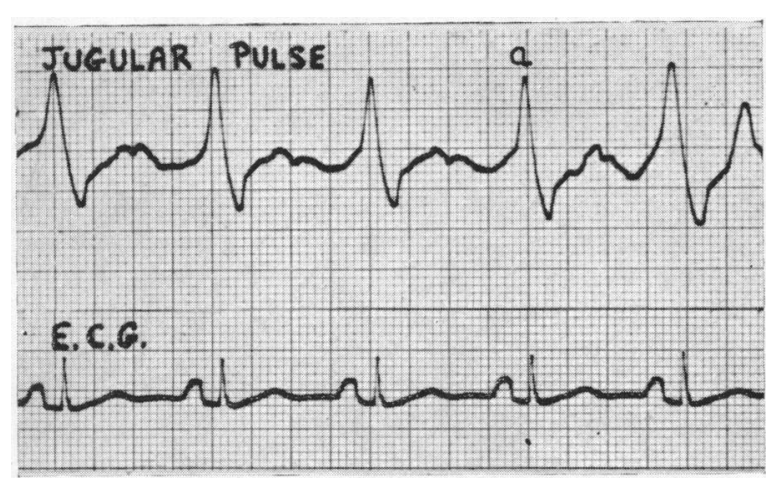

Fig. 1.-Jugular phlebogram from a case of tricuspid stenosis with normal rhythm, showing giant $a$ waves.

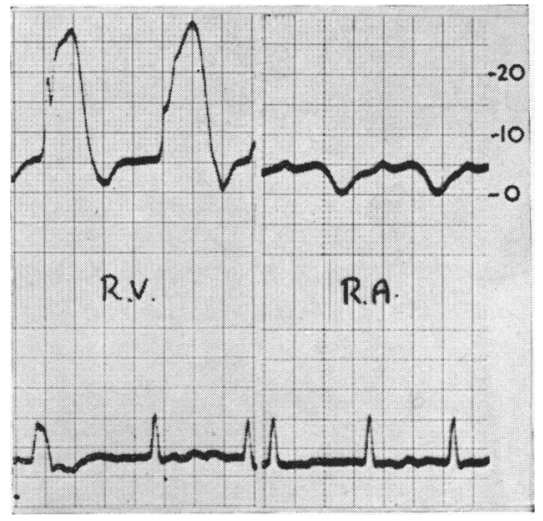

Fig. 2.-Right atrial and right ventricular pressure pulses from a case of constrictive pericarditis, showing a steep $y$ descent, dominant $y$ trough, and rapid equalization of diastolic pressures. 
steep, right atrial and right ventricular diastolic pressures equalizing with the opening of the tricuspid valve at the summit of $v$, and falling together to the trough $y$, which is the lowest pressure reached by the relaxing right ventricle (Fig. 2). Following $y$ right atrial and right ventricular diastolic pressures rise sharply together, as further filling is resisted by the distended right ventricle so that a conspicuous $y$ trough is formed. In constrictive pericarditis the ascent following $y$ is especially sharp, because the rigid pericardium prevents further filling relatively early in diastole; the situation is similar in tense pericardial effusion, and not very different in advanced heart failure with or without tricuspid incompetence when the right ventricle is overloaded. In tricuspid stenosis, however, right atrial and right ventricular diastolic pressures do not equalize at the summit of $v$ when the tricuspid valve opens, the obstruction causing a pressure gradient across the valve as the ventricular pressure falls in early diastole. The right atrial pressure declines more gradually, and since the right ventricle offers no resistance to filling, there is no sharp ascent from the $y$ nadir; indeed, right atrial and right ventricular diastolic pressures may not equalize at all in severe cases. The whole effect on the venous pulse is to produce a relatively gentle $y$ descent with little or no rebound which can be recognized at the bedside (Fig. 3). This observation alone led to a clinical diagnosis of tricuspid stenosis in several of our cases. The same kind of pressure pulse may be recorded from the left atrium in cases of mitral stenosis (Owen and Wood, 1955).

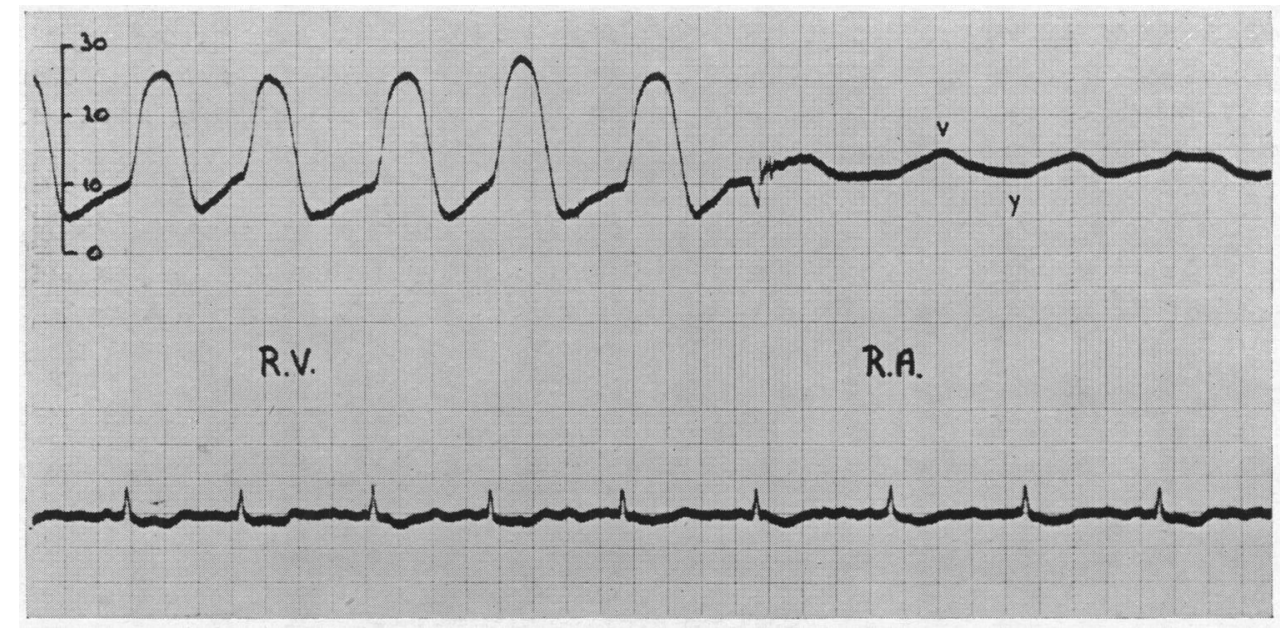

FIG. 3.-Right atrial and ventricular pressure pulses from a case of tricuspid stenosis with auricular fibrillation, showing a gentle $y$ descent, insignificant $y$ trough, and slow equalization of diastolic pressures.

Pulse. Normal rhythm was present in eight cases and auricular fibrillation in six. The pulse was small and firm in nine cases, water-hammer in quality in the three with aortic incompetence, and anacrotic in the two with aortic stenosis.

Colour. Peripheral cyanosis was observed in nine cases, presumably due to vasoconstriction associated with a low cardiac output. Neither jaundice nor excessive pigmentation was noticeable.

Cardiac Impulse. The characteristic palpatory sign of tricuspid stenosis was absence of the right ventricular heave usually felt in cases of mitral stenosis with a high pulmonary vascular resistance. The absence of this sign is, of course, in harmony with uncomplicated mitral stenosis, but in such cases, the venous pressure is normal.

Heart Sounds and Murmurs. On deep inspiration accentuation of the first heart sound and of the opening snap at the tricuspid area was observed in two of the three cases with dominant tricuspid stenosis.

A presystolic or diastolic murmur, waxing on inspiration, was heard over the tricuspid area in 
all but one case (Fig. 4). Although this physical sign has long been recognized (Duroziez, 1868), it may easily be overlooked unless due care is taken. A tricuspid systolic murmur, also maximal on inspiration, was heard in only four instances, and was attributed to associated tricuspid incompetence. Auscultatory signs of mitral valve disease (stenosis in all but one) were always present in addition.

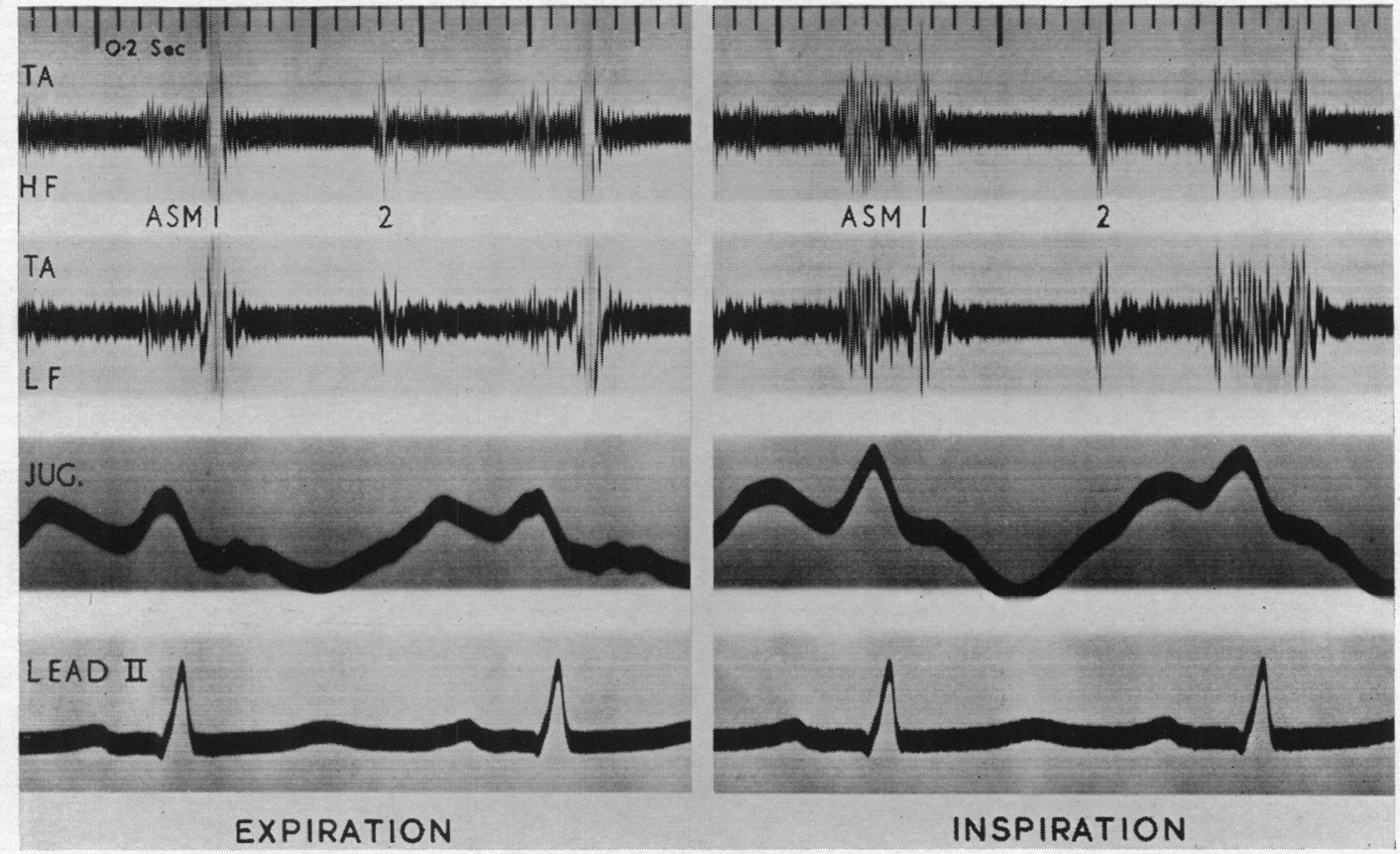

FIG. 4.-Phonocardiogram from the tricuspid area in a case of tricuspid stenosis, showing waxing of the presystolic murmur (ASM) on inspiration. Both records were taken with the same degree of amplification.

The Electrocardiogram. The characteristic feature of the electrocardiogram was a tall right atrial $P$ wave, measuring more than $2.5 \mathrm{~mm}$. in height, in the absence of electrocardiographic evidence of considerable right ventricular hypertrophy (Fig. 5), and as such it was observed in seven of the eight cases with normal rhythm, the mean height of the $P$ wave being $3.7 \mathrm{~mm}$.; the exception was the mildest case in the series. Unlike the pure $\mathbf{P}$ pulmonale, the $\mathbf{P}$ wave of tricuspid stenosis is nearly always widened owing to delay in its left atrial component due to concomitant mitral stenosis.

Electrocardiographic evidence of moderate right ventricular hypertrophy was present in three cases, two of which had a moderately raised pulmonary vascular resistance and one pulmonary stenosis. Left ventricular hypertrophy was found in four cases, all of which had aortic valve disease.

\section{RADIOLOGY}

Dressler and Fischer (1929) emphasized that enlargement of the heart to the right in the absence of pulmonary venous congestion was strong evidence of tricuspid stenosis. The most characteristic feature in our series was conspicuous dilatation of the right atrium without enlargement of the pulmonary artery (Fig. 6), this combination being observed in 11 out of the 14 cases; of the 3 exceptions two had enlargement of the pulmonary artery due to coincident pulmonary hypertension secondary to mitral stenosis, and one had pulmonary valve stenosis with post-stenotic dilatation of the pulmonary artery. Pulmonary venous congestion was absent or slight in ten, the tricuspid 


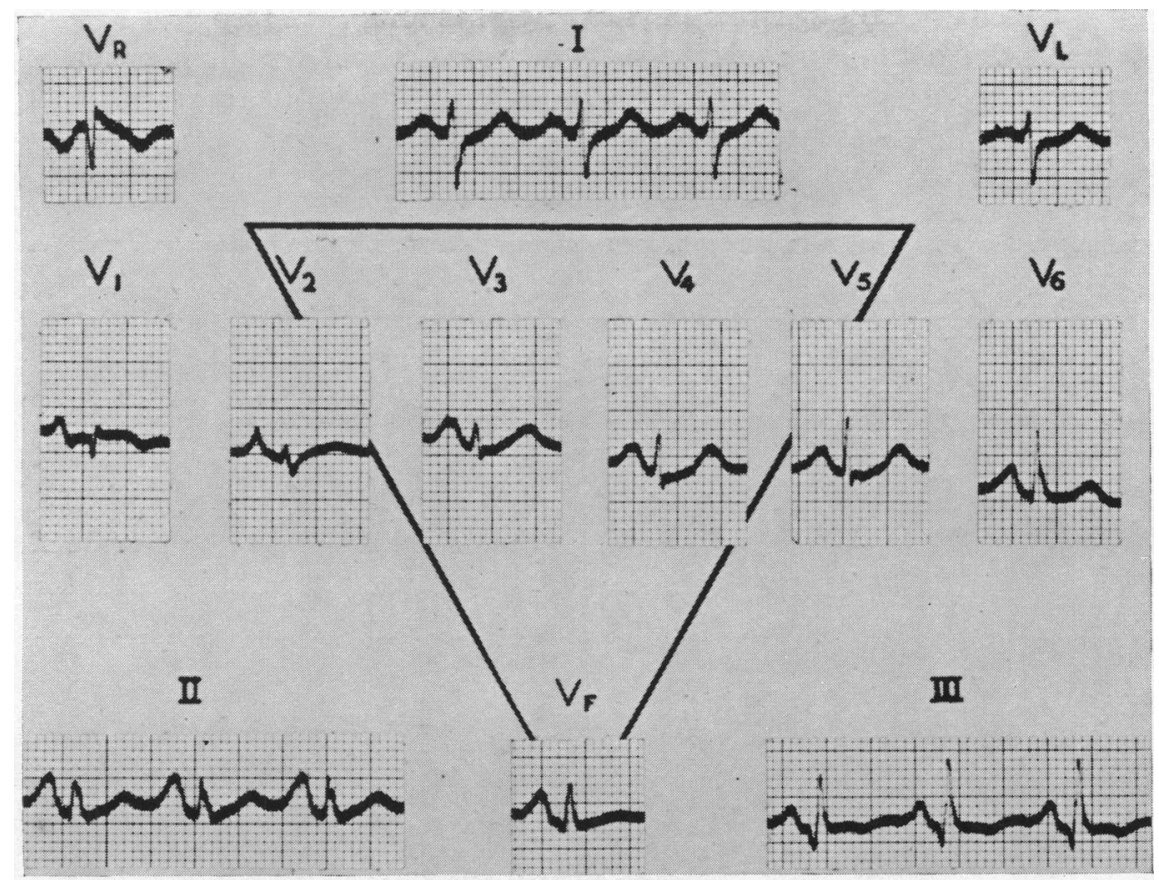

FIG. 5.-Electrocardiogram from a case of tricuspid stenosis with normal rhythm, showing tall right atrial $P$ waves and no evidence of right ventricular hypertrophy.

stenosis tending to lower the cardiac output; but it was considerable in four cases with severe mitral stenosis requiring surgical relief. Dilatation of the left atrium was moderate in 11 and slight in 3 cases. A prolonged search for calcification of the tricuspid valve was fruitless in all instances.

\section{ISOLATED TRICUSPID STENOSIS}

A case of disseminated lupus erythematosus with isolated tricuspid stenosis is included in this series, because as far as we are aware, this is the first time such a lesion has been recognized clinically. Of 23 fatal cases described by Baehr et al. (1935), a non-rheumatic verrucous endocarditis of the mitral or tricuspid valve was observed in thirteen. Gross (1940) found obvious macroscopic involvement of the tricuspid valve in 6 of his 23 cases. Bagenstoss (1952) states that an abacterial verrucous endocarditis occurs in 40 per cent of all cases and that it is found with equal frequency on both sides of the heart. In their series of 34 cases Shearn and Pirofsky (1952) observed an apical diastolic murmur in four; in two of these necropsy was performed and revealed Libman-Sachs endocarditis of the mitral valve in each case.

Case Report. A man, aged 33, previously in good health, had been ill for one year with irregular fever. effort dyspnœa, and recurrent hæmoptyses. A systolic murmur was heard at the left sternal edge, but there was no anæmia, petechiæ, nor systemic emboli; the sputum was negative for tubercle bacilli, repeated blood cultures were sterile, and he did not improve following prolonged antibiotic therapy. Serial skiagrams showed right atrial dilatation, slight cardiac enlargement, transient lung shadows, and persistent enlargement of a right hilar gland (Fig. 7). On examination, his general condition was quite good, but he had a low-grade fever and sinus tachycardia; the venous pressure was raised and showed $a$ and $v$ waves of equal amplitude $5 \mathrm{~cm}$. above the sternal angle at $45^{\circ}$; at the left sternal edge there was a presystolic murmur and an early systolic murmur, clearly maximal on inspiration. The erythrocyte sedimentation rate (Westergren) was $17 \mathrm{~mm}$. in one hour, and there was a polymorphonuclear leucocytosis, but no LE cells in the peripheral blood. The electrocardiogram was normal. On cardiac catheterization an 8-mm. $\mathrm{Hg}$. diastolic pressure gradient across the tricuspid valve was demonstrated and the indirect left atrial pressure was normal. There was subjective improvement following prolonged steroid therapy, and the skiagram showed a diminution 
in the size of the right hilar gland as well as disappearance of the right apical lesion, but the heart murmurs and the venous pressure and pulse remained unchanged.

In this case, there was no clinical nor physiological evidence of any other valve lesion and a diagnosis of subacute disseminated lupus erythematosus with isolated tricuspid stenosis was made.

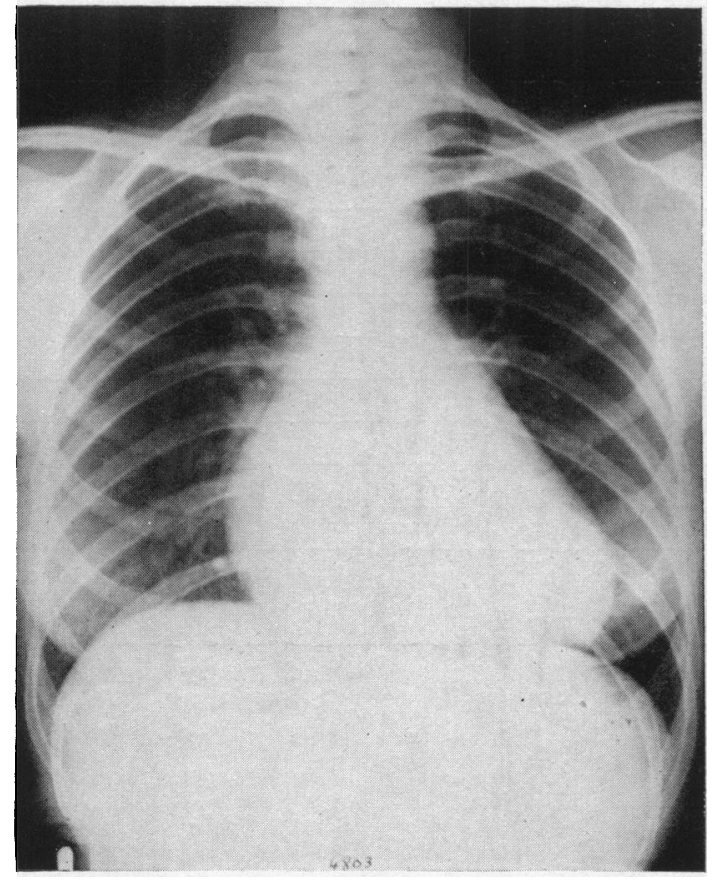

FIG. 6.-Skiagram showing dilatation of the right atrium without enlargement of the pulmonary artery and without pulmonary venous congestion in a case of dominant tricuspid stenosis.

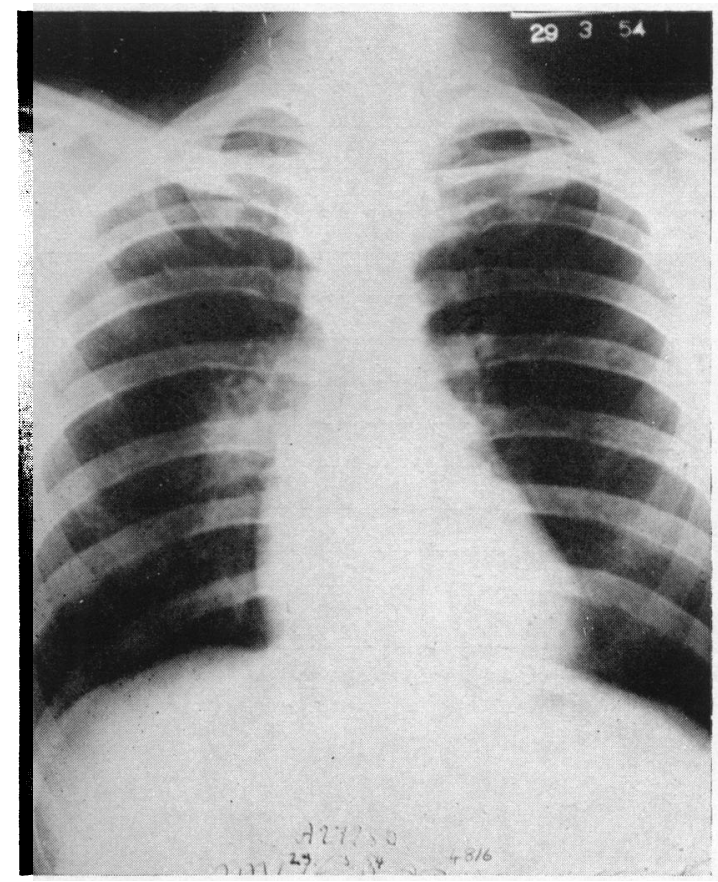

FIG. 7.-Skiagram from a case of isolated tricuspid stenosis, probably due to disseminated lupus erythematosus, showing enlargement of right hilar glands and slight right atrial dilatation.

\section{CATHETER Studies}

A diastolic pressure gradient across the tricuspid valve has been described by Gorlin and Gorlin (1951) and by Ferrer et al. (1953). Twelve of the fifteen cases in this series were investigated by means of cardiac catheterization and the results are shown in the Table. Pressures were recorded with a Sanborn electromanometer and polyviso, using the sternal angle as reference point. Oxygen consumption was measured with a Sanborn metabulator and blood gas analysis on the Van Slyke apparatus.

In each case the diagnosis was proved by the demonstration of a diastolic or presystolic pressure gradient, averaging $6 \mathrm{~mm}$. $\mathrm{Hg}$, across the tricuspid valve. This is best seen when the intracardiac pressure is recorded continuously as the catheter is withdrawn from the right ventricle to the right atrium; normally right atrial and ventricular diastolic pressures are identical (Fig. 8). The gradient has been measured as the greatest difference between right atrial and ventricular pressures during ventricular diastole. With normal rhythm the maximum gradient is presystolic (Fig. 9) and with auricular fibrillation it is early diastolic (Fig. 10).

The pulmonary vascular resistance was normal in all but two cases in which it was moderately increased. It was measured as mean P.A.P. minus mean L.A.P. (indirect) divided by Cardiac output (litres a minute). The cardiac output was less than 4 litres/min. in eight cases. In three instances there was considerable elevation of the left atrial pressure due to mitral stenosis which required mitral valvotomy. 
TABLE I

Results of CATHeter Studifs

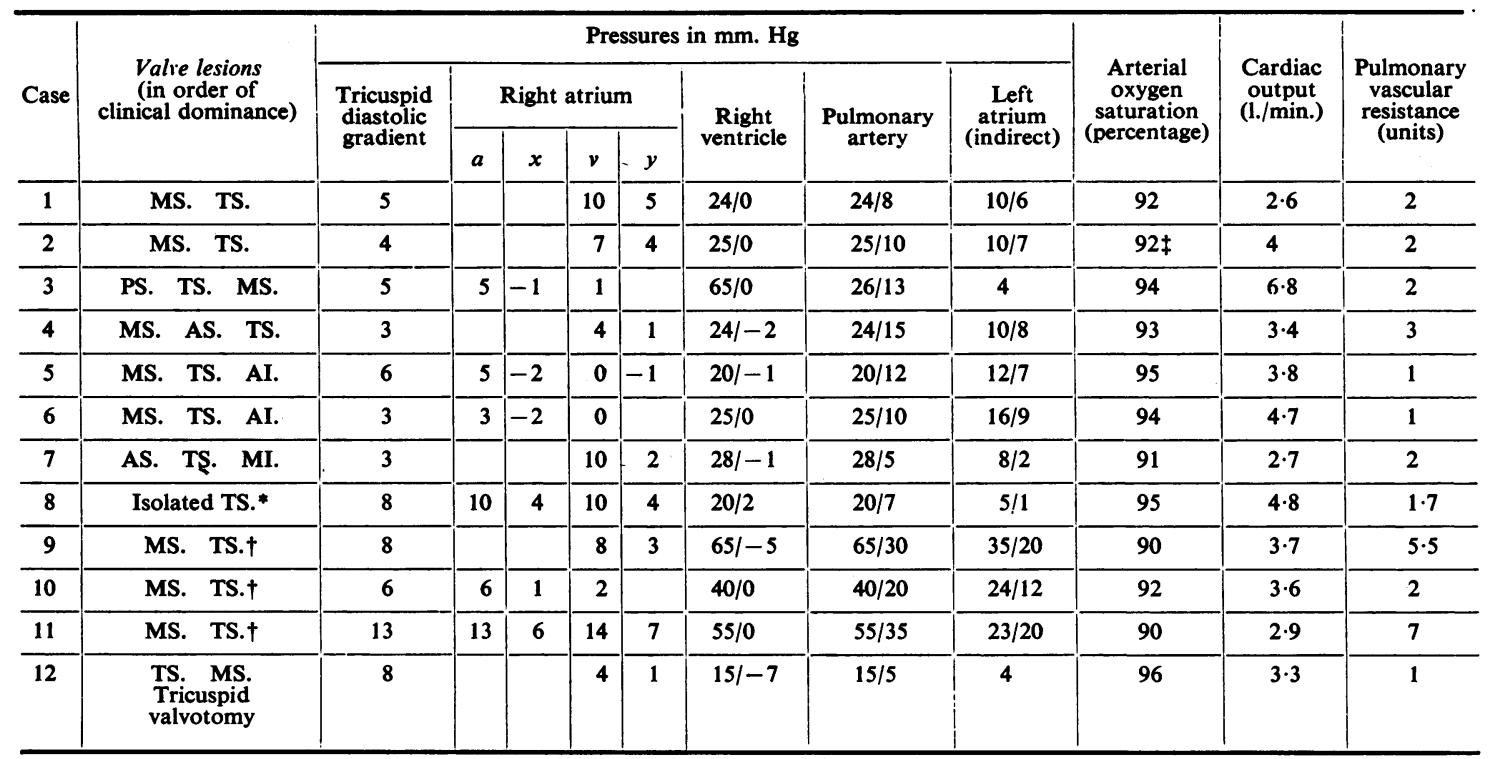

* Case 8 had disseminated lupus erythematosus. † These patients have had mitral valvotomy. $\ddagger=$ assumed. TS $=$ Tricuspid stenosis. MS = Mitral stenosis. $\mathbf{A S}=$ Aortic stenosis. $\mathbf{A I}=$ Aortic incompetence. $\mathbf{P S}=$ Pulmonary stenosis.

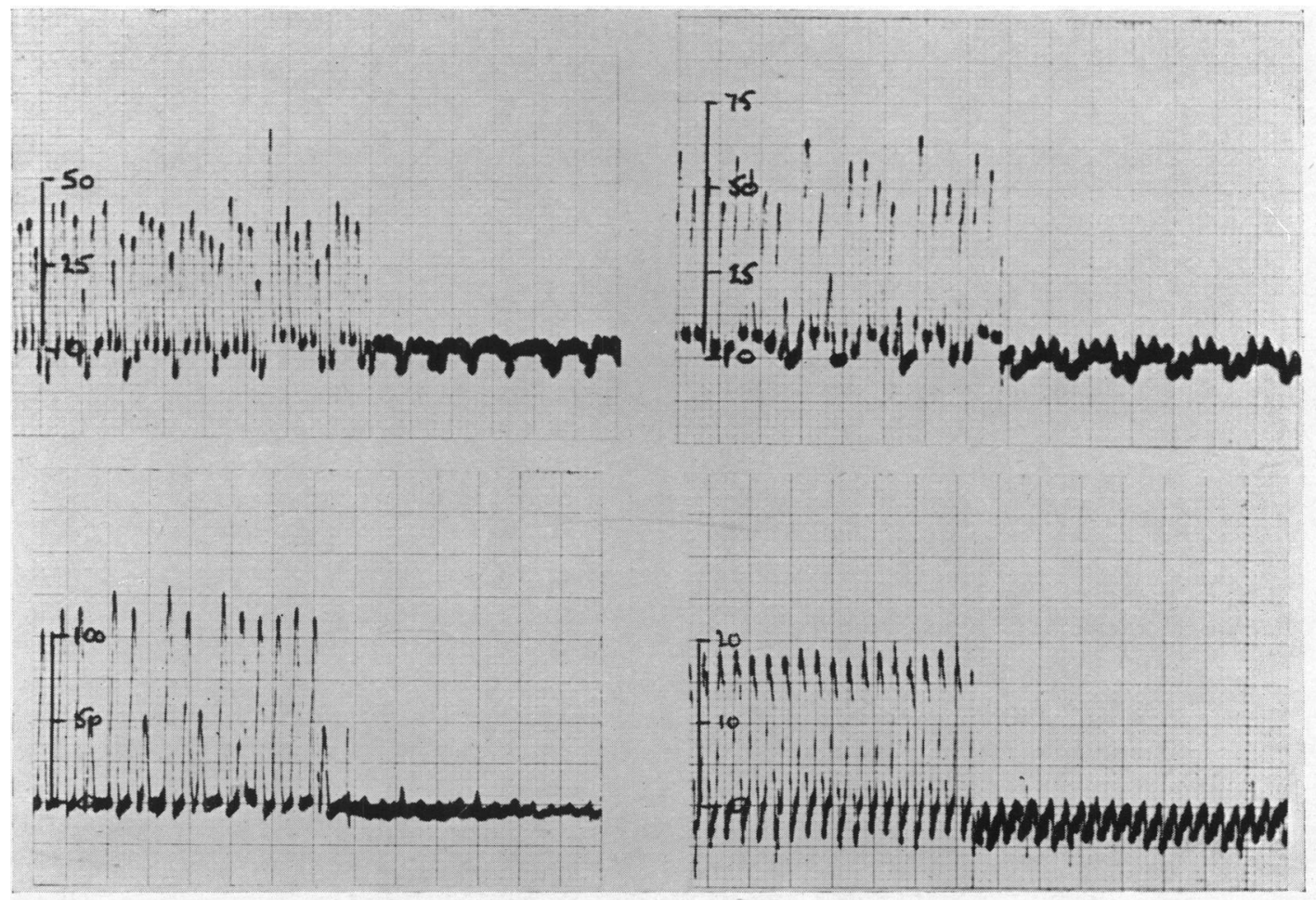

FIG. 8.-Pressure tracings during withdrawal of the catheter from right ventricle to right atrium in four cases of uncomplicated mitral stenosis showing that right atrial and ventricular diastolic pressures are normally identical. 


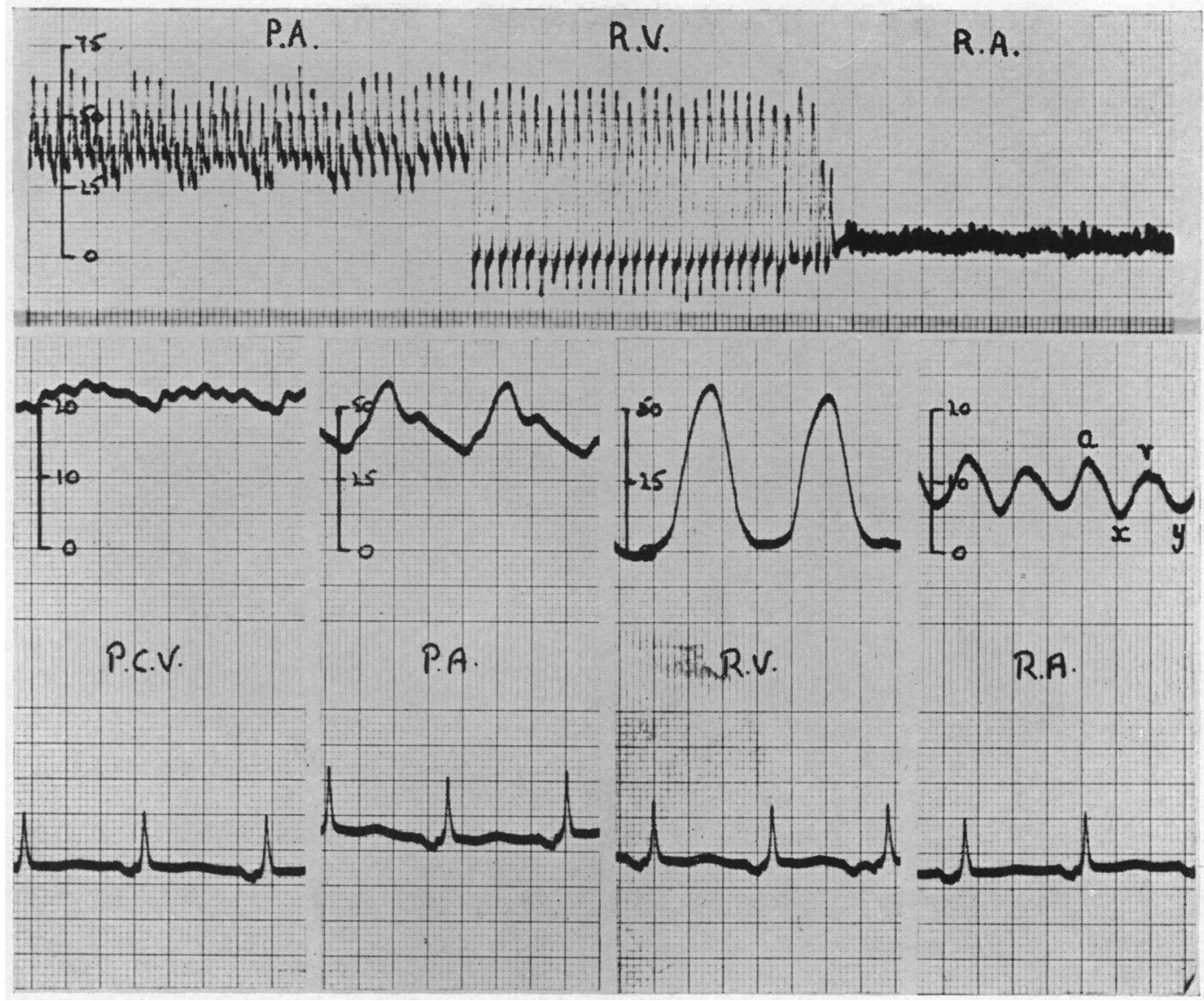

FIG. 9.-Right atrial and right ventricular pressure pulses and withdrawal tracing from a case of tricupid stenosis with normal rhythm, showing a $9 \mathrm{~mm}$. Hg presystolic pressure gradient across the tricuspid valve.

In one case with dominant tricuspid stenosis, the most severe in the series, tricuspid valvotomy was performed by Sir Russell Brock. Pressures recorded before valvotomy demonstrated a diastolic pressure gradient across the tricuspid valve, a gentle $y$ descent despite the high venous pressure, absence of a properly defined $y$ trough, and very delayed equalization of right atrial and ventricular diastolic pressures. After valvotomy, right ventricular filling was obviously much more rapid, as indicated by the fairly steep $y$ descent, clear $y$ trough, and sharp rise in right ventricular diastolic pressure, resulting in virtual elimination of the diastolic pressure gradient at mid-diastole (Fig. 11).

\section{Discussion}

A confident bedside diagnosis of tricuspid stenosis should not be difficult if the condition is borne in mind, and in fact was made in 80 per cent of this small series. Its 3 per cent frequency is based on a carefully analysed group of 300 cases of mitral valve disease, in 199 of which continuous intracardiac pressure tracings were obtained as the catheter was withdrawn from the right ventricle to the right atrium. Again, during the period in which we have been studying tricuspid stenosis, similar withdrawal tracings have been obtained in a total of 270 cases of mitral valve disease, of which only 11 had a diastolic pressure gradient across the tricuspid valve, giving a relative incidence of 4.1 per cent for tricuspid stenosis amongst all the mitral cases catheterized. The first figure is 


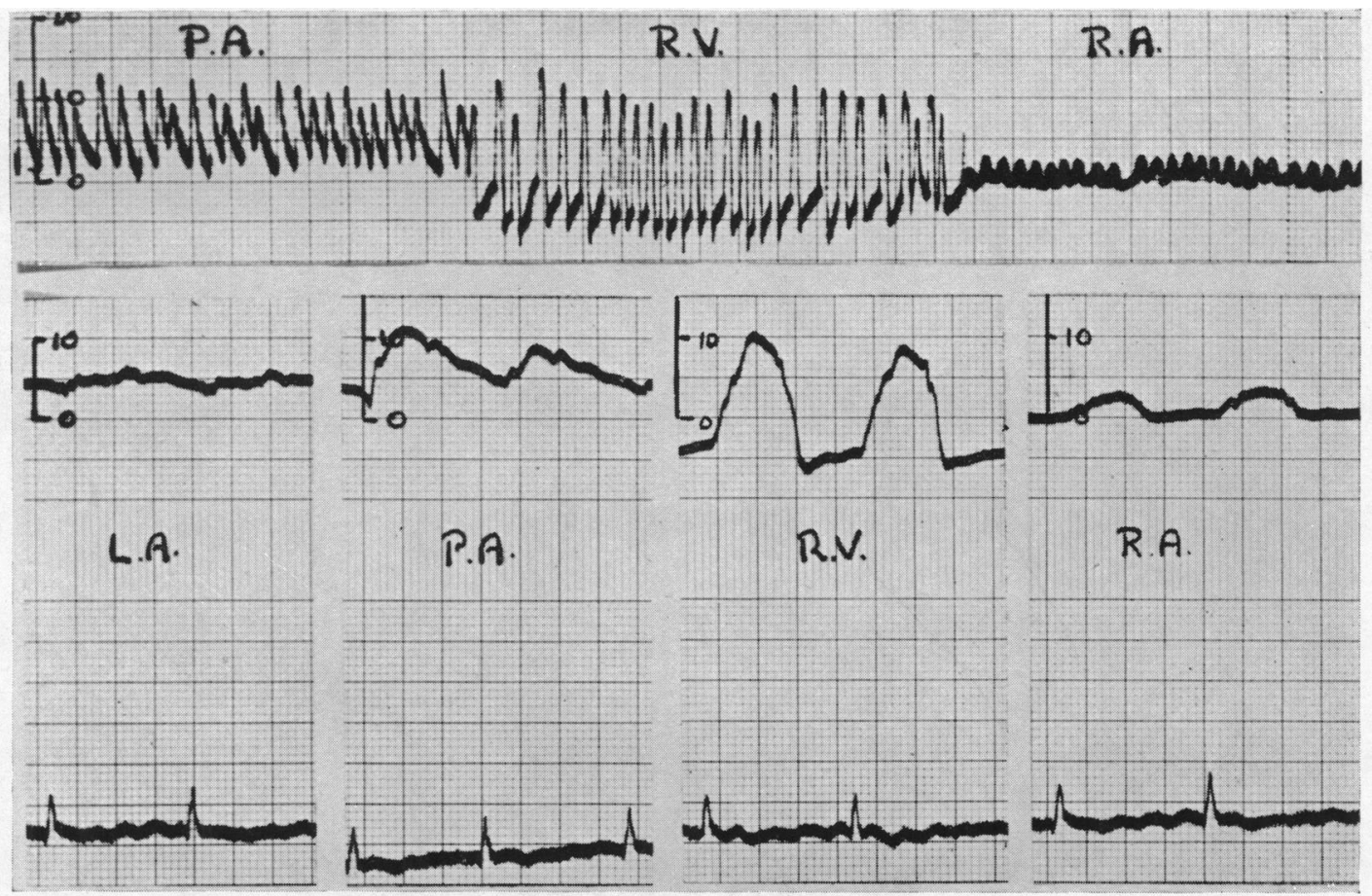

FIG. 10.-Right atrial and right ventricular pressure pulses and withdrawal tracing from a case of tricuspid stenosis with auricular fibrillation, showing a $5 \mathrm{~mm}$. $\mathrm{Hg}$ diastolic pressure gradient across the tricuspid valve.

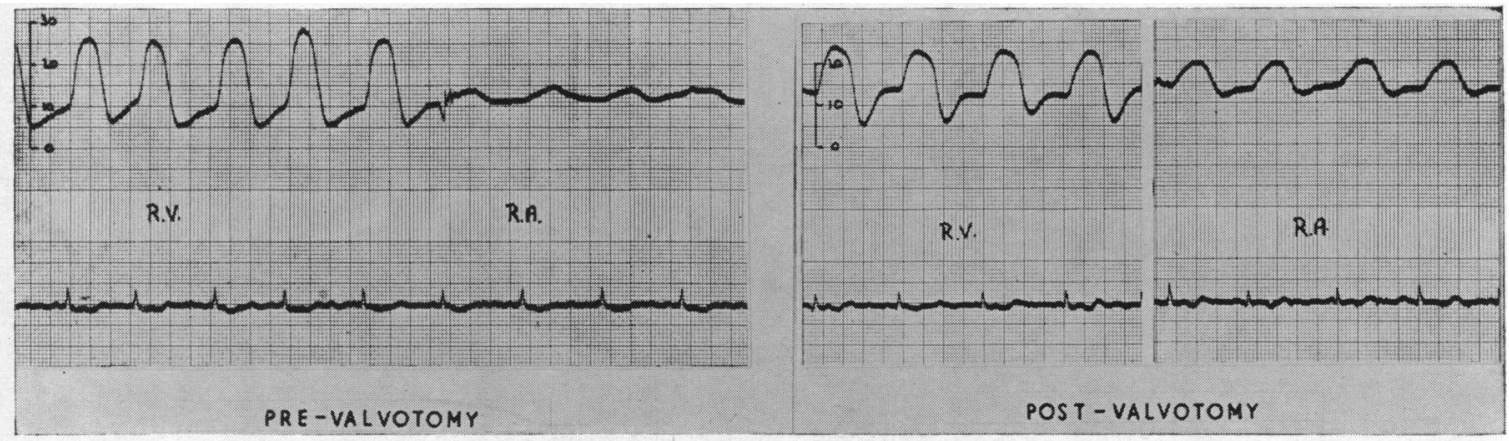

Fig. 11.-Right atrial and right ventricular pressure pulses in a case of tricuspid stenosis before and after tricuspid valvotomy, showing the change in the form of the $y$ descent, the elimination of the diastolic pressure gradient across the tricuspid valve, and rapid filling of the right ventricle after valvotomy.

probably nearer the truth, however, because catheterization was more likely to have been carried out if tricuspid stenosis was suspected on clinical grounds.

In practice, the possibility of tricuspid stenosis should be considered in any case of mitral valve disease in which the jugular venous pressure is unquestionably raised. The differential diagnosis then lies between uncontrolled auricular fibrillation, severe mitral incompetence, a high pulmonary vascular resistance, pericardial effusion, and tricuspid stenosis, for in uncomplicated mitral stenosis the venous pressure is normal (Wood, 1954). If the rise in venous pressure is due to a rapid irregular ventricular rate, it soon subsides in response to digitalis therapy. 
The raised venous pressure that may be associated with severe mitral incompetence is not yet fully understood, but it may be due primarily to left ventricular failure, leading to a diminished renal blood flow, sodium retention, and hydræmia; or it may be due to a filling defect of the right ventricle from the bulged interventricular septum (Bernheim effect). We have never encountered tricuspid stenosis in the presence of severe mitral incompetence, so if the latter is present there is little point in looking further for the cause of the raised venous pressure.

If a high pulmonary vascular resistance is responsible for the abnormal jugular venous pressure pulse, there is likely to be a giant $a$ wave with normal rhythm or a steep $y$ descent and conspicuous $y$ trough with auricular fibrillation; while the latter combination at once excludes tricuspid stenosis, the former, of course, does not. The chief clinical features of severe pulmonary hypertension are a heaving right ventricle, damped mitral auscultatory signs, loud pulmonary valve closure or functional pulmonary incompetence, strong right ventricular preponderance electrocardiographically, and considerable dilatation of the pulmonary artery radiologically. When any of these features are present, tricuspid stenosis can only be diagnosed at the bedside if there is an unequivocal inspiratory tricuspid presystolic or diastolic murmur. A separate tricuspid opening snap, accentuated during inspiration, may be heard occasionally, but is usually unconvincing unless the mitral valve is heavily calcified when -its tricuspid origin may be assumed with confidence. Unfortunately, only one of the fifteen cases of tricuspid stenosis in this series had calcific mitral stenosis, so we have little information to offer concerning isolated tricuspid snaps. The exception had trivalvular disease and was too complicated to be helpful.

The differential diagnosis between tricuspid stenosis and pericardial effusion may sometimes be difficult, for in both conditions there is a high venous pressure and apparent dilatation of the right atrium without evidence of advanced pulmonary hypertension. However, the jugular pulse has no specially characteristic feature in pericardial effusion, $a$ and $v$ waves being of more or less equal amplitude in cases with normal rhythm, and the $y$ descent being in harmony with the height of $v$ in cases with auricular fibrillation. If necessary, the presence or absence of pericardial effusion can be proved during cardiac catheterization by demonstrating whether or not the heart shadow lies beyond the right atrial border as defined by the tip of a suitably looped catheter (Wood, 1950).

Isolated tricuspid stenosis of rheumatic origin has not been described, although Clements (1935) reported a case as such despite clear necropsy signs of associated mitral valve disease. In the case of isolated tricuspid stenosis in our series, probably due to Libman-Sachs endocarditis, the clinical features of the tricuspid lesion itself were identical with those of the rheumatic cases.

\section{SUMMARY}

A series of fourteen cases of rheumatic tricuspid stenosis is presented. The lesion was found in 3 to 4 per cent of all cases of mitral valve disease, but was overshadowed by the latter in all but three instances.

A case of probable disseminated lupus erythematosus with isolated tricuspid stenosis is also reported.

The clinical features of tricuspid stenosis are described and include the following.

(1) Absence of symptoms of pulmonary venous congestion: chronic œdema and ascites are only seen in advanced cases.

(2) A high venous pressure in the absence of a high pulmonary vascular resistance, pericardial effusion, uncontrolled auricular fibrillation, or severe mitral incompetence.

(3) A venous pulse showing a giant $a$ wave in cases with normal rhythm, and a gentle $y$ descent without a properly defined $y$ trough in those with auricular fibrillation.

(4) A quiet right ventricle and unimpressive pulmonary second sound.

(5) A diastolic or presystolic murmur at the tricuspid area, waxing on inspiration.

(6) An opening snap at the left sternal edge in the presence of calcific mitral stenosis.

(7) A tall right atrial $P$ wave in the absence of electrocardiographic evidence of right ventricular hypertrophy. 
(8) Conspicuous dilatation of the right atrium without enlargement of the pulmonary artery, and with relatively little pulmonary venous congestion.

The diagnosis was proved in twelve cases during cardiac catheterization by demonstrating a presystolic or diastolic pressure gradient across the tricuspid valve, averaging $6 \mathrm{~mm}$. $\mathrm{Hg}$.

Of the fifteen cases, twelve were diagnosed initially at the bedside by means of these criteria, and three were discovered during cardiac catheterization.

We should like to thank the cardiological technicians at the Brompton Hospital and the National Heart Hospital, particularly Mrs. Milne and Mr. Brabrook-Norman, for their invaluable help. We are also indebted to Mr. D. F. Kemp of the Photographic Department, the Institute of Diseases of the Chest, for the illustrations.

\section{REFERENCES}

Baehr, G., Klemperer, P., and Schifrin, A. (1935). Trans. Ass. Amer. Phys., 50, 139.

Bagenstoss, A. H. (1952). Proc. Mayo. Clin., 27, 412.

Bramwell, U. C., and King, J. T. (1942). The Principles and Practice of Cardiology. Oxford University Press, London. Clements, A. B. (1953). Amer. J. med. Sci., 190, 389.

Cooke, W. T., and White, P. D. (1941). Brit. Heart J., 3, 147.

Dressler, W., and Fischer, R. (1929). Klin. Wschr., 8, 1267.

Duroziez, P. L. (1868). Gaz. Hôp., Paris, 310.

Friedberg, C. K. (1949). Diseases of the Heart. W. B. Saunders, Philadelphia.

Ferrer, M. I., Rejane, M., Harvey, M., Kuschner, M., Richards, D. W., and Cournand, A. (1953). Circulation Res., 1, 49.

Gorlin, R., and Gorlin, S. G. (1951). Amer. Heart J., 41, 1.

Gross, L. (1940). Amer. J. Path., 16, 375.

Kossman, C. E. (1955). Circulation, 11, 378.

Mackenzie, J. (1902). The Study of the Pulse. Edinburgh, Young J. Pentland.

Owen, S. G., and Wood, P. (1955). Brit. Heart J., 17, 41.

Shearn, M. A., and Pirofsky, B. (1952). Arch. intern. Med., 90, 790.

Smith, J. A., and Levine, S. A. (1942). Amer. Heart J., 23, 739.

Thompson, P. T., and Levine, S. A. (1937). Amer. J. med. Sci., 193, 4.

White, P. D. (1951). Heart Disease. 4th ed., New York, Macmillan Co.

Wood, P. (1950). Proc. R. Soc. Med., 43, 195.

(1954). Brit. med. J., 1, 1051, and 1113. 